\title{
ANÁLISIS DE LA RESPUESTA DE OCHO ESPECIES NATIUAS DEL BOSQUE ALTO ANDINO ANTE DOS MÉTODOS DE PROPAGACIÓN'
}

Palabras clave: Propagación sexual, especies nativas, bosque alto andino.

Key words: Sexual spread, vegetative spread, native species, high Andean forest.

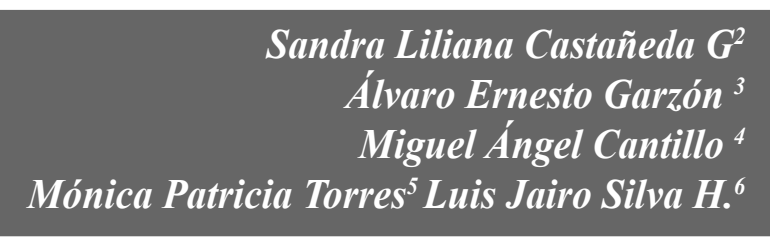

\section{RESUMEN}

Esta investigación se enfocó en obtener información acerca de las especies nativas que de acuerdo a sus características dinamogenéticas son una alternativa en los procesos de restauración ecológica de los bosques altos andinos colombianos. Se escogieron y se propagaron las especies Baccharis latifolia (R \& P), Bocconia frutescens L., Cordia cylindrostachya (R \& P), Diplostephium rosmarinifolium (Benth), Drymis granadensis L f., Eupatorium angustifolium (Kunth), Palicourea vaginata Benth, y Palicourea linearifolia Wernham. Estas especies cuentan con una descripción morfológica de flores, frutos y semillas, junto con los resultados de las pruebas ISTA. El establecimiento de ensayos de propagación se realizó en los viveros de la Universidad Distrital y el Parque La Florida, contempló cómo tratamientos en la propagación sexual cuatro concentraciones de giberelina (GA3) y tres intensidades de sombra, mientras que en la propagación vegetativa se manejaron dos diámetros y cinco concentraciones de ácido indol-3 butírico (AIB). Los resultados mostraron que las especies Bocconia frutescens y Palicourea vaginata requieren de tratamientos pregerminativos en las semillas, para aumentar la velocidad y el porcentaje de germinación. Por otro lado, las semillas de Baccharis latifolia, Diplostephium rosmarinifolium, Drymis granadensis, Eupatorium angustifolium y Palicourea vaginata tienen características fotoblásticas, por lo cual necesitan de sombra para una germinación adecuada. Las especies Cordia cylindrostachya y Palicourea linearifolia muestran graves problemas en su propagación sexual debido al ataque por insectos del orden Lepidoptera e Hymenoptera en sus semillas. Respecto al comportamiento de todas especies ante los tratamientos de AIB, se observa que no existe una concentración particular que favorezca su desarrollo, sino que la combinación entre las concentraciones de AIB y el diámetro resultó en una respuesta diferente para cada una de las especies de

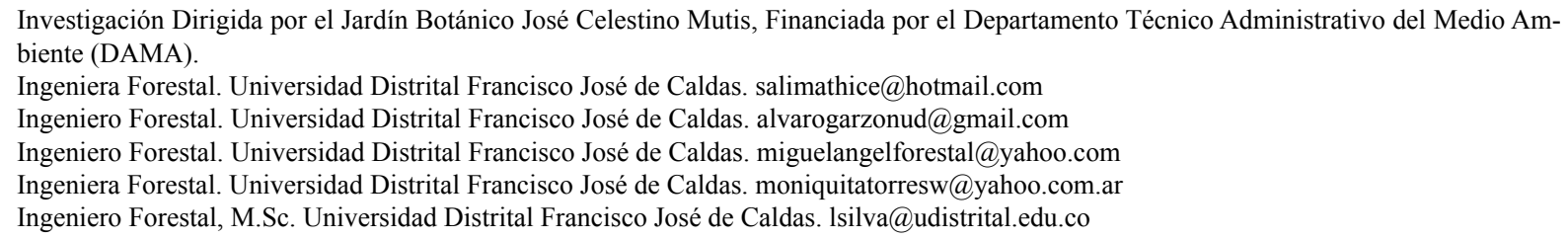


estudio. Adicionalmente, con relación a la escala de propagación asexual las especies Bocconia frutescens, Cordia cylindrostachya, Palicourea vaginata, Diplostephium rosmarinifolium y Drymis granadensis se encontró que son muy difíciles de reproducir.

\section{ABSTRACT}

The objective of this research was to generate information on the native tree species represent an alternative in the ecological restoration of the Colombian high Andean forests, according to its dynamo-genetic characteristics. We have chosen and spread the species: Baccharis latifolia (R \& P), Bocconia frutescens L., Cordia cylindrostachya (R \& $\mathrm{P})$, Diplostephium rosmarinifolium (Benth), Drymis granadensis L f., Eupatorium angustifolium (Kunth), Palicourea vaginata Benth, and Palicourea linearifolia Wernham. The species include a morphological description of flowers, fruits and seeds, and ISTA tests. The spread experiments were made in the nurseries of the Universidad Distrital and La Florida park. For the sexual spread, we have used as treatments four gibberellins concentrations and three shadow conditions, while the vegetative spread consisted of two diameters and five indol butiric acid (IBA) concentrations. Results have shown that pre-germination treatments are needed for Bocconia frutescens y Palicourea vaginata, in order to increase the probability and germination rate. On the other hand, shadow conditions are needed for Baccharis latifolia, Diplostephium rosmarinifolium, Drymis granadensis, Eupatorium angustifolium and Palicourea vaginata, as their seeds exhibit photoblastic characteristics. Due to the Cordia cylindrostachya and Palicourea linearifolia seed attack by insects (Lepidoptera and Hymenoptera) their sexual spread is highly limited. Finally, regarding the species response to the IBA and diameter combinations, each species responded in a different manner. Additionally, regarding the vegetative spread, the species Bocconia frutescens, Cordia cylindrostachya, Palicourea vaginata, Diplostephium rosmarinifolium and Drymis granadensis were very difficult to spread.

\section{INTRODUCCIÓN}

La escasa información acerca de la reproducción de especies nativas con fines de restauración ecológica, y la necesidad de desarrollar metodologías de producción que garanticen la obtención del material vegetal necesario para recuperar la estructura, funcionalidad y autosuficiencia de un ecosistema que ha sido degradado, dieron origen a una investigación acerca de la propagación de ocho especies nativas del bosque Alto Andino.

Desde hace algunos años, el Jardín Botánico de Bogotá, José Celestino Mutis en conjunto con el Departamento Administrativo del Medio Ambiente (DAMA) desarrollaron tres líneas de investigación en las cuales la propagación sexual y asexual de especies nativas construía un eslabón importante en el proceso de restauración ecológica que se lleva a cabo en los Cerros Orientales del Distrito Capital. Igualmente, diferentes instituciones, centros de investigación, universidades y organizaciones no gubernamentales han dedicado recursos para indagar acerca de la reproducción de especies nativas con el objetivo de suplir el déficit de información acerca de las mismas. Por ejemplo, autores como Muñetones y Garcés (2000), García (2001), Reyes (2001) y Guevara (2001) entre otros, han estudiado la propagación de especies nativas de importancia botánica y económica a nivel nacional.

Por lo tanto, con el fin de contribuir a los procesos de restauración ecológica del bosque alto andino del Distrito Capital, se analizó la respuesta de de ocho especies nativas ante dos métodos de propagación (propagación sexual 
y asexual). El proceso incluyó la aplicación de hormonas vegetales como la giberelina (GA3) y el ácido indol-3 butírico (AIB), debido al conocimiento sobre sus efectos y la efectividad que presentan al estimular el adecuado desarrollo de los individuos. Las hipótesis planteadas hacen referencia al efecto que genera la aplicación de las hormonas en el desarrollo de los individuos, con base en los factores de concentración, tratamiento e interacciones presentes.

\section{METODOLOGÍA}

\section{SELECCIÓN E IDENTIFICACIÓN DE LAS ESPECIES EN ESTUDIO}

Dentro del convenio celebrado entre el Jardín Botánico y el Departamento Técnico Administrativo del Medio Ambiente (DAMA) se esco- gieron ocho especies de importancia ecológica según sus atributos y su distribución natural en el bosque alto Andino, para propagar sexual y asexualmente, y emplear posteriormente en procesos de restauración ecológica en los Cerros Orientales. Las siguientes son las especies en mención (Tabla 1):

La revisión preliminar de información botánica y taxonómica, junto con la identificación de especies asociadas y fitogeografía, permitió identificar las especies de estudio y determinar los lugares de procedencia. Una vez se inspeccionaron las reservas forestales de San Cristóbal, La Aguadora y San Francisco con el fin de localizar los árboles pertenecientes a una comunidad -sin problemas fitosanitarios y que no estuvieran aislados- se obtuvo el material vegetal necesario para la propagación.

Tabla 1. Listado de especies en estudio.

\begin{tabular}{|l|c|c|c|}
\hline \multicolumn{1}{|c|}{ Nombre común } & Nombre científico & Familia & Hábito \\
\hline Chilco & Baccharis latifolia $(\mathrm{R} \& \mathrm{P})$ & Asteraceae & Arbusto \\
Trompeto & Bocconia frutescens L. & Papaveraceae & Árbol \\
Salvio & Cordia cylindrostachya $(\mathrm{R} \& \mathrm{P})$ & Boraginaceae & Árbol \\
Romero blanco & Diplostephium rosmarinifolium (Benth) & Asteraceae & Arbusto \\
Canelo de páramo & Drymis granadensis Lf. & Winteraceae & Árbol \\
Blanquillo & Eupatorium angustifolium (Kunth) & Asteraceae & Arbusto \\
Tominejero & Palicourea vaginata Benth & Rubiaceae & Árbol \\
Tominejero & Palicourea linearifolia Wernham & Rubiaceae & Árbol \\
\hline
\end{tabular}

\section{OBTENCIÓN DEL MATERIAL VEGETAL} Y MANEJO

En esta etapa, de los árboles padres se cosecharon los frutos maduros manualmente de las copas. Se tomaron muestras de árboles maduros y sin problemas fitosanitarios, para realizar posteriormente su beneficio. En el caso de las especies Drymis granadensis L. f., Diplostephium rosmarinifolium, Cordia cylindrostachya, y Palicourea linearifolia, fue necesario el ascenso a los mismos para poder cosechar los frutos, debido a su porte.

Se realizó una descripción morfológica de flores, frutos y semillas, siguiendo la guía para caracterizar e identificar semillas propuesta por Aníbal Niembro (1992). Los frutos recolectados se clasificaron de acuerdo a sus características morfológicas, y su beneficio varió dependiendo del tipo del mismo (Ver anexo 1). Después de

Revista Colombia Forestal Vol. 10 No. 20 - Diciembre 2007 
realizar el beneficio, las semillas se dejaron al aire libre para ser secadas durante una semana; luego se empacaron en frascos herméticos y se llevaron a la nevera a una temperatura de $4^{\circ} \mathrm{C}$, según el procedimiento común. Adicionalmente, se realizaron las pruebas de determinación de la calidad física y fisiológica de acuerdo con los principios del análisis de semillas normalizado por Internacional Seed Test Association (ISTA).

Para la propagación vegetativa de las especies se tomaron brotes ortotrópicos sanos y vigorosos, presentes en la sección basal del árbol. Las estacas fueron defoliadas, humedecidas y empacadas en bolsas de polietileno para evitar la desecación en el proceso de transporte. Al día siguiente se llevaron al lugar de plantación en donde se clasificaron en dos grupos teniendo en cuenta el diámetro. En cada estaca se dejó en lo posible igual número de yemas -por lo menos tres- y se les realizó un corte en bisel en la parte inferior con el fin de aumentar el área de influencia de la auxina ácido indol-3 butírico (AIB).

\section{MONTAJE DE LOS ENSAYOS DE PROPAGACIÓN}

Los ensayos de propagación se montaron siguiendo el procedimiento convencional, ajustando algunos detalles según las características de las especies para establecer finalmente los ensayos de propagación en el vivero de la Universidad Distrital con una réplica del montaje de estacas en el vivero del Parque La Florida.

Para el establecimiento de los ensayos de propagación sexual se tomó un total de cincuenta (50) semillas por unidad experimental en el caso de las especies Baccharis latifolia, Eupatorium angustifolium y Diplostephium rosmarinifolium. Para las otras especies se tomó un valor mínimo de 15 semillas por unidad experimental, previa escarificación de las aquellas que lo requerían. En el caso de las semillas de las especies Eupatorium angustifolium, Baccharis latifolia, Diplostephium rosmarinifolium y Drimys granadensis que no presentan inconvenientes en la permeabilidad de su cubierta seminal, se efectúo un diseño experimental en bloques completos al azar, considerando como bloques tres porcentajes de sombra $(0,30$ y $60 \%$ ). Diseño que tuvo en cuenta como tratamientos cuatro concentraciones de Giberelina GA3 $(0,1000,2000$ y 4000).

Para la especie Palicourea vaginata se utilizó el mismo diseño experimental. Sin embargo, a esta especie por la tener una semilla de testa dura e impermeable se le realizó un proceso de escarificación química con acido sulfúrico en una concentración del $30 \%$ por un tiempo de 10 minutos. En el caso de la especie Bocconia frutescens que presenta problemas respecto a la permeabilidad de su cubierta seminal se empleó un diseño experimental completamente al azar con arreglo factorial de $3 * 4$, en el cual se tuvieron en cuenta tres concentraciones de Giberelina GA3 $(0,2000$ y 4000 ppm) y cuatro tipos de escarificación (sin escarificación, mecánica, agua caliente, agua caliente-fría). Conociendo las características de la especie fue necesario el uso una polisombra del $30 \%$ para todo el diseño experimental.

Finalmente para el caso de la especie Cordia cylindrostachya, por la baja disponibilidad de semillas se utilizó un diseño experimental completamente al azar, con cuatro concentraciones de giberelina GA3 (0, 1000, 2000 y 4000 ppm) como tratamientos; además se realizó una escarificación con ácido sulfúrico al $10 \%$ por un tiempo de 20 minutos.

Los datos se registraron diariamente por un periodo de 4 meses a partir de la siembra de las semillas y se obtuvieron en términos del número total de semillas germinadas por tratamiento. Con los datos de semillas germinadas diaria- 
mente se obtuvo el valor de la germinación y con el último registro se realizó el análisis de varianza para el porcentaje de germinación.

Para la propagación vegetativa se tomaron como factores dos diámetros de estaca $(\theta>\mathrm{y}$ $\theta<)$ y cinco concentraciones de ácido indol-3 butírico (AIB) (0, 1000, 2000, 4000 y 8000). Se evaluaron las variables número de rebrotes, porcentaje de sobrevivencia, longitud del rebrote más largo y porcentaje de enraizamiento. Las observaciones se realizaron semanalmente y al momento de realizar el levantamiento del ensayo (4 meses como mínimo). Se tomó el número de estacas enraizadas por tratamiento haciendo uso de la escala de propagación basada en trabajos de Quesada y Gutiérrez (1972) y adaptada por Segura et al. (1991) para determinar una calificación acerca de la propagación vegetativa de cada especie.

\section{SEGUIMIENTO, CONTROL Y OBTENCIÓN DE RESULTADOS}

El seguimiento se realizó diariamente mediante observaciones, toma de datos, riego y control sanitario de los ensayos experimentales. Se obtuvieron los datos de acuerdo al ensayo de propagación respectivo y sus variables, para posteriormente realizar el análisis estadístico pertinente al diseño experimental. Este análisis se realizó por medio del programa SPSS, verificando el cumplimiento de los criterios estadísticos de homogeneidad de varianzas, normalidad y aleatoriedad a través de la prueba de Levene. También se realizó la comparación entre residuales estudentizados y esperados por medio de la prueba no paramétrica de Rachas. Los datos se transformaron en los casos en los que alguno de los criterios no se cumpliera, y se efectuó el análisis de varianza (univariante) con un nivel de confianza del 95\%. Se determinaron las diferencias significativas en los tratamientos empleados por medio del Test de Tukey, obteniendo finalmente el trata- miento efectivo de propagación para cada una de las especies.

\section{RESULTADOS}

\section{PRUEBAS DE CALIDAD FÍSICA}

Tras la realización de las pruebas ISTA, se observa que en general las especies presentan un porcentaje de pureza significativo y favorable para trabajar, exhibiendo valores altos o cercanos al $50 \%$, contrario al valor reportado para la especie Diplostephium rosmarinifolium que tiene una gran cantidad de impurezas al colectar su semilla. Con relación al contenido de humedad $(\mathrm{CH})$ los valores varían dependiendo de la especie. No obstante, se podría decir que las especies de la familia Asteraceae muestran valores similares por tener semillas desnudas de forma aspergiliforme que no favorecen la retención de humedad en su interior. En contraste, tanto especies como Bocconia frutescens, Cordia cylindrostachya, Drimys granadensis y Palicourea vaginata, con contenidos de humedad disímiles, poseen testas y endospermos diferentes (forma y tamaño) condicionando la permeabilidad de su semilla y por ende el grado de humedad que presentan.

Respecto al número de semillas por kilogramo las especies de la familia Asteraceae presentan los valores más altos debido al tamaño tan pequeño de sus semillas, contrario a las demás especies que presentan valores altos pero que difieren en número de una a otra. Ahora bien, con relación a la viabilidad al corte y en tetrazolio, se nota que en general, aunque los valores varían entre especies, que se pueden reproducir por semilla gracias a la calidad de su endospermo y embrión. No obstante, la especie Cordia cylindrostachya muestra limitaciones para reproducirse sexualmente debido a la calidad de su semilla. Al analizar el porcentaje de germinación controlada muestra que las especies Eupatorium angustifolium, Baccharis

Revista Colombia Forestal Vol. 10 No. 20 - Diciembre 2007 
latifolia, Bocconia frutescens y Diplostephium rosmarinifolium, podrían desarrollarse adecuadamente en vivero, mientras que las especies Cordia cylindrostachya, Drimys granadensis y Palicourea vaginata están seriamente com- prometidas. Además, Palicourea linearifolia Benth no reporta resultados debido a la presencia de larvas que consumen por completo el endospermo de la semilla. En la Tabla 2 se observan los resultados obtenidos por especie.

Tabla 2. Resultados de calidad física de las especies de estudio.

\begin{tabular}{|c|c|c|c|c|c|c|c|c|c|}
\hline \multirow{2}{*}{\multicolumn{2}{|c|}{ ESPECIE }} & \multirow{2}{*}{\multicolumn{2}{|c|}{ PROCEDENCIA }} & \multicolumn{6}{|c|}{ CALIDAD FÍSICA } \\
\hline & & & & $\mathrm{P}(\%)$ & $\mathrm{CH}(\%)$ & Sem/Kg & VC\% & VT\% & G\% \\
\hline \multicolumn{2}{|c|}{ Baccharis latifolia } & 1 & & 79.90 & 17,50 & $27 ’ 874.564$ & 53,12 & 46,43 & 32,14 \\
\hline \multicolumn{2}{|c|}{ Bocconia frutescens } & 1 & & 99,00 & 7,80 & 48.200 & 98,31 & 56,18 & 26,11 \\
\hline \multicolumn{2}{|c|}{ Cordia cylindrostachya } & $\|$ & & 67,90 & 59,10 & 38.377 & 23,14 & 10,05 & - \\
\hline \multicolumn{2}{|c|}{ Diplostephium rosmarinifolium } & III & & 28,70 & 17,80 & $5 ’ 111.821$ & 30,24 & 28,27 & 17,00 \\
\hline \multicolumn{2}{|c|}{ Drimys granadensis } & III & & 91,00 & 47,70 & 209.556 & 69,36 & 77,23 & 2,01 \\
\hline \multicolumn{2}{|c|}{ Eupatorium angustifolium } & IV & & 47,00 & 23,50 & 4'403.538 & 40,04 & 38,16 & 32,32 \\
\hline \multicolumn{2}{|c|}{ Palicourea linearifolia } & V & & \multicolumn{6}{|c|}{$\begin{array}{l}\text { No se reportan resultados por la presencia de larvas que } \\
\text { consumen por completo el endospermo de la semilla }\end{array}$} \\
\hline \multicolumn{2}{|c|}{ Palicourea vaginata } & $\mathrm{VI}$ & & 46,42 & 13,70 & 44.385 & 40,13 & - & - \\
\hline \multicolumn{10}{|c|}{ Donde: } \\
\hline I & Reserva forestal de La Aguadora & $P$ & \multicolumn{7}{|c|}{ Pureza } \\
\hline II & Municipio de Gachalá (Cundinamarca) & $\mathrm{CH} \%$ & \multicolumn{7}{|c|}{ Contenido de Humedad \% } \\
\hline III & Reserva forestal de San Francisco & Sem $/ \mathrm{Kg}$ & \multicolumn{7}{|c|}{ Semillas/kg } \\
\hline IV & Reserva forestal de San Cristóbal & VC & \multicolumn{7}{|c|}{ Viabilidad Corte } \\
\hline V & $\begin{array}{l}\text { Reserva Forestal La Aguadora, } \\
\text { Parque Entre Nubes y Laguna de Pedro Palo }\end{array}$ & VT & \multicolumn{7}{|c|}{ Viabilidad Tetrazolio } \\
\hline $\mathrm{VI}$ & Reserva forestal Parque Entre Nubes & G & \multicolumn{7}{|c|}{ Germinación } \\
\hline
\end{tabular}

\section{ENSAYOS DE PROPAGACIÓN SEXUAL}

Con base en el análisis descriptivo y estadístico de los resultados de la propagación sexual, se observa que las especies Bocconia frutescens, Diplostephium rosmarinifolium, Eupatorium angustifolium se desarrollan mejor bajo la aplicación de giberelina, mientras que Baccharis latifolia responde mejor sin la hormona. El desarrollo de los individuos de Drymis granadensis y Palicourea vaginata no responde de manera significativa a ninguna concentración.
Se observa que porcentajes de sombra del $30 \%$ y $60 \%$ son favorables para el desarrollo de las especies Baccharis latifolia, Diplostephium rosmarinifolium, Drymis granadensis y Eupatorium angustifolium, en tanto que para Bocconia frutescens su desarrollo se favorece cuando la semilla se escarifica mecánicamente y se trata sin hormona, mientras que al escarificarla solamente la longitud plantular es mejor. Esto tiene implicaciones si los que se busca es alguna de estas características en particular. Por su parte, las especies Cordia cylindrostachya y Palicourea linearifolia no se pudieron 
propagar sexualmente debido a que se encontró la presencia de insectos en el interior de la semilla, los cuales comprometen seriamente su reproducción al consumir el endospermo de la misma. Estos insectos pertenecen a las familias Notodóntidae y Gelichiidae (Orden Lepidoptera) y Eulóphidae (Orden Hymenoptera). A continuación se observan los resultados detallados en la Tabla 3.

Tabla 3. Resultados estadísticos de la propagación sexual de las especies de estudio.

\begin{tabular}{|l|c|c|}
\hline \multirow{2}{*}{ Especie } & \multicolumn{2}{|c|}{ Propagación Sexual } \\
\cline { 2 - 3 } & Tratamiento & Bloque / Factor* $^{*}$ \\
\hline Baccharis latifolia & $0 \mathrm{ppm}$ & Sombra $30 \%$ \\
Bocconia frutescens. & $2000 \mathrm{ppm}$ & Testigo - Escarificación mecánica (Sobrevivencia) \\
& $2000 \mathrm{ppm}$ \\
Cordia cylindrostachya & Nula: Presencia de insectos - Flias & (Notodón mecánica (Longitud plantular) y Gelichiidae - LEPIDOP.) \\
Diplostephium rosmarinifolium & 2000 y $4000 \mathrm{ppm}$ & Sombra 60\% \\
Drymis granadensis & NS & Sombra 30\% \\
Eupatorium angustifolium & NS - Mejor: $1000 \mathrm{ppm}$ & Sombra 30\% \\
Palicourea linearifolia & Nula: Presencia de insectos - Flia & (Eulóphidae - HYMENOP.) \\
Palicourea vaginata & NS & Sombra 30\% \\
\hline
\end{tabular}

NS: No significativo

En general, las especies de la familia Asteraceae presentaron un crecimiento que en número favorecería su reproducción pero con el paso del tiempo, no todas logran sobrevivir. La especie Eupatorium angustifolium es la que mejor se desarrolla y responde ante la aplicación de giberelina, pues las plántulas exhiben un tamaño mayor y un mejor crecimiento. La especie Baccharis latifolia responde bien sin aplicar la hormona pero se tarda un poco más en alcanzar altura y establecerse en el medio aunque en número su germinación sea notoria. En tanto que Diplostephium rosmarinifolium responde a la aplicación de giberelina pero en diferente grado pues el número de plántulas es menor y requiere un mayor porcentaje de sombra.

La especie Drimys granadensis aunque tarda más tiempo en germinar muestra buena sobrevivencia, así como desarrollo progresivo y logra establecerse con el paso del tiempo bajo un porcentaje de sombra intermedio, mientras que Bocconia frutescens es una especie que se desarrolla muy bien y alcanza alturas considerables para cortos periodos de tiempo, pero que necesita ser tratada cuidadosamente porque es una especie que al transplantar presenta una alto porcentaje de mortalidad, aspecto que debe ser estudiado con detenimiento posteriormente.

\section{ENSAYOS DE PROPAGACIÓN ASEXUAL}

Al realizar el análisis estadístico de los resultados de la propagación asexual se encontró que los factores evaluados para varias especies no tienen resultados significativos estadísticamente, pero con el seguimiento del desarrollo de cada una de las especies se notaron que existen tratamientos ante los cuales las especies responden mejor. 
Con relación a los factores número de rebrotes, sobrevivencia, longitud del rebrote más largo y enraizamiento, se observa que las especies que desarrollaron un mayor número de rebrotes fueron Baccharis latifolia y Drymis granadensis seguidas por Cordia cylindrostachya, Eupatorium angustifolium, Bocconia frutescens y Palicourea linearifolia, mientras que Palicourea vaginata y Diplostephium rosmarinifolium presentaron los menores valores de número de rebrotes por periodo de tiempo.

El porcentaje de sobrevivencia obtenido finalmente en los ensayos de propagación no muestra valores altos (excepto Bocconia frutescens), aspecto que deja ver que es preciso identificar el momento en el cual las especies están en mejores condiciones de desarrollo para ser transplantadas definitivamente y cuidar su desarrollo sin comprometer su sobrevivencia. En este factor los valores para cada una de las especies varían mostrando los valores más bajos las especies Diplostephium rosmarinifolium, Palicourea linearifolia y Palicourea vaginata.

Ahora bien, respecto a la longitud de los rebrotes se aprecia que los rebrotes más largos los presenta Baccharis latifolia seguida de Bocconia frutescens, mientras que las demás especies presentan rebrotes de longitud intermedia, excepto Diplostephium rosmarinifolium cuyos retoños son muy pequeños. Se observa que no existe un tratamiento en particular ante el cual las especies reaccionan mejor, sino que cada una responde de manera variada. No obstante, se encontró que bajo una concentración de 4000 ppm ninguna especie responde mejor respecto al número de rebrotes, sobrevivencia, longitud del rebrote más largo y enraizamiento, aspecto que permitiría entonces no recomendar su uso.

En el proceso se percibe que en diámetros mayores, las estacas responden de manera fa- vorable. Los porcentajes de enraizamiento dependen de cada especie porque no todas logran desarrollar raíces como es el caso de Diplostephium rosmarinifolium y Palicourea vaginata, cuya propagación no sería factible por este medio. Por otra parte y como característica particular, se observa que la especie Drymis granadensis es la única que desarrolla cayo en sus estacas y la mejor concentración para que esto suceda es la de 2000 ppm en un diámetro menor. Los resultados se aprecian claramente en la Tabla 4.

Finalmente, al analizar los resultados del análisis de varianza de los ensayos de propagación asexual se nota que los valores no son significativos estadísticamente para las variables de número de rebrotes, sobrevivencia, longitud del rebrote más largo y enraizamiento. No obstante, a las especies Bocconia frutescens, Diplostephium rosmarinifolium y Palicourea vaginata no se les pudo realizar el análisis estadístico por la tasa de mortalidad que presentaron. Esto permite sugerir que estas especies no responden favorablemente ante este tipo de propagación. Ahora bien, con respecto a la escala de propagación propuesta por Quesada \& Gutiérrez (1972) y adaptada por Segura et al. (1991), se tiene que la especie Baccharis latifolia se propaga de difícil a moderadamente fácil, Palicourea linearifolia de difícil a muy difícil y Eupatorium angustifolium difícilmente se puede reproducir por este medio. En tanto que las especies Bocconia frutescens, Cordia cylindrostachya, Diplostephium rosmarinifolium, Drymis granadensis L. f y Palicourea vaginata, presentan una propagación muy difícil, considerando la respuesta que éstas dieron a la aplicación de tratamientos hormonales para inducir su desarrollo. Los resultados se observan claramente en la Tabla 5. 
Tabla 4. Resultados descriptivos de la propagación asexual para las especies de estudio.

\begin{tabular}{|c|c|c|c|c|c|c|c|c|c|c|c|c|c|}
\hline & Especie & NTR & Días* & $\% \mathbf{S}$ & $\begin{array}{l}\text { LRL } \\
(\mathrm{cm})\end{array}$ & $\begin{array}{c}\text { Tto } \\
\text { (ppm) }\end{array}$ & $\theta$ & $\% \mathrm{E}$ & $\begin{array}{c}\text { Tto } \\
\text { (ppm) }\end{array}$ & $\theta$ & $\% \mathrm{C}$ & $\begin{array}{c}\text { Tto } \\
\text { (ppm) }\end{array}$ & $\theta$ \\
\hline \multicolumn{2}{|c|}{ Baccharis latifolia } & 1037 & 30 & 22 & 45 & 2000 & $>$ & 35 & 1000 & $<$ & - & & - \\
\hline \multicolumn{2}{|c|}{ Bocconia frutescens } & 260 & 29 & 74 & 29 & 8000 & $<$ & ** & 8000 & $<$ & - & - & - \\
\hline \multirow{2}{*}{\multicolumn{2}{|c|}{ Cordia cylindrostachya }} & 502 & 39 & 37 & 13 & 8000 & $>$ & 7 & 0 & $<$ & - & - & - \\
\hline & & & & & & & & 7 & 1000 & $>$ & - & - & - \\
\hline \multicolumn{2}{|c|}{ Diplostephium rosmarinifolium } & 74 & 44 & 11 & 0,6 & 0 & $>$ & - & & - & - & - & - \\
\hline \multicolumn{2}{|c|}{ Drymis granadensis $L . f$} & 952 & 75 & 38 & 5 & 2000 & $>$ & 7 & 2000 & $>$ & 14 & 2000 & $<$ \\
\hline \multicolumn{2}{|c|}{ Eupatorium angustifolium } & 390 & 34 & 53 & 12 & 1000 & $>$ & 30 & 8000 & $>$ & - & - & - \\
\hline \multicolumn{2}{|c|}{ Palicourea linearifolia } & 222 & 37 & 17 & 9 & 0 & $<$ & 14 & 4000 & $>$ & - & - & - \\
\hline \multicolumn{2}{|c|}{ Palicourea vaginata } & 103 & 90 & 27 & 3 & 2000 & $<$ & - & - & - & - & - & - \\
\hline \multicolumn{14}{|l|}{ Donde } \\
\hline NTR & \multicolumn{13}{|l|}{ № total de rebrotes } \\
\hline$\% S$ & \multicolumn{13}{|c|}{$\%$ de sobrevivencia } \\
\hline LRL & \multicolumn{13}{|c|}{ Longitud del rebrote más largo } \\
\hline Tto (ppm) & \multicolumn{13}{|l|}{ Tratamiento (ppm) } \\
\hline$\theta$ & \multicolumn{13}{|l|}{ Diámetro } \\
\hline & \multicolumn{13}{|l|}{$\%$ de enraizamiento } \\
\hline & \multicolumn{13}{|c|}{ \% de callogénesis } \\
\hline \multicolumn{14}{|c|}{ *Tiempo en el que se presentó el mayor número de rebrotes } \\
\hline ** Tan solc & o una estaca desarrol & raíz (4 & & & & & & & & & & & \\
\hline
\end{tabular}

Tabla 5. Resultados estadísticos de la propagación asexual para las especies de estudio.

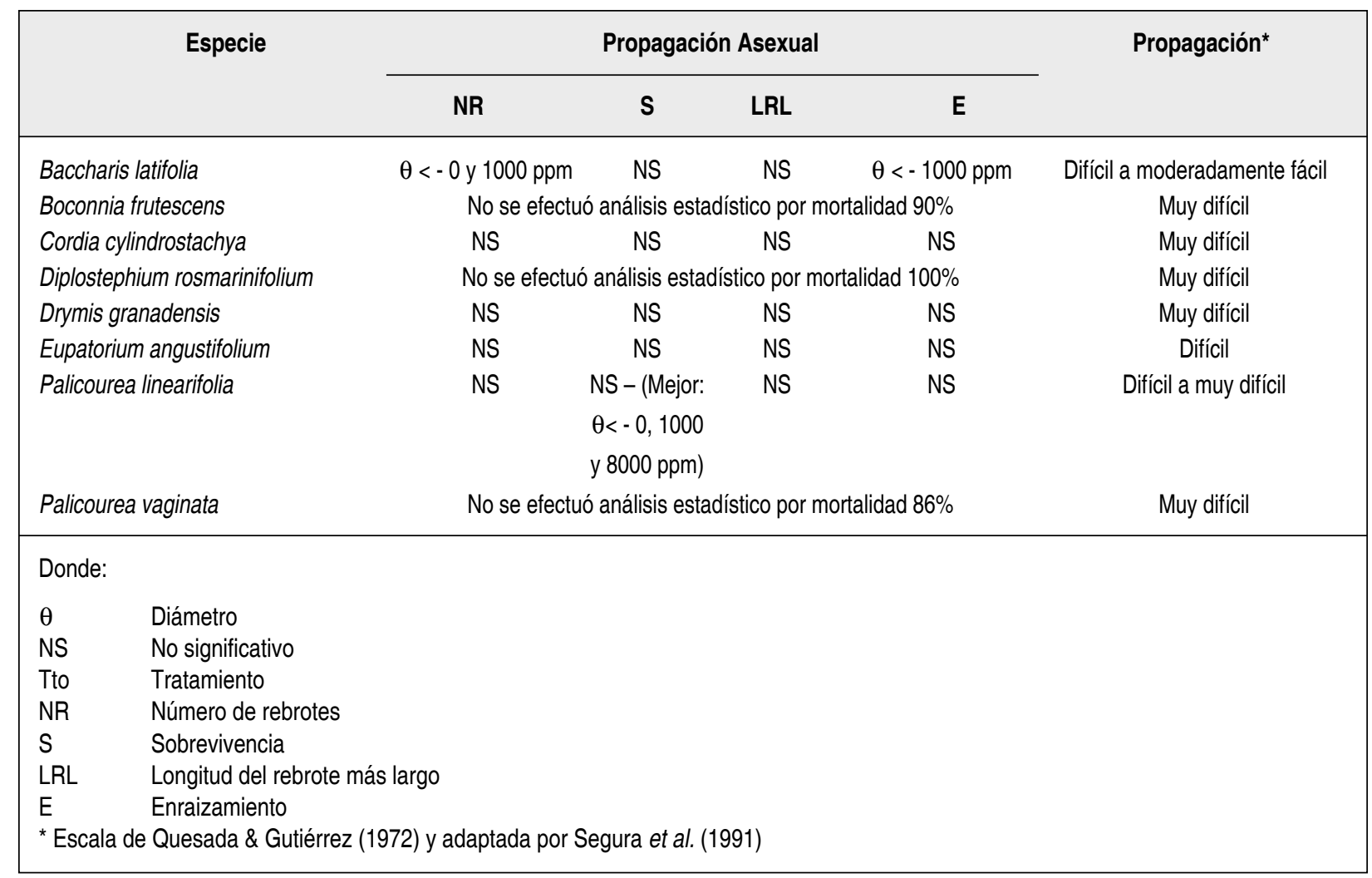




\section{CONCLUSIONES}

Las semillas de Baccharis latifolia, Diplostephium rosmarinifolium, Drimys granadensis, Eupatorium angustifolium y de Palicourea vaginata tienen características fotoblásticas, es decir, que para una adecuada germinación de las plántulas, las semillas requieren de sombra, aproximadamente entre $30 \%$ y $60 \%$ la cual permita el estímulo lumínico para el adecuado desarrollo del embrión.

Para las semillas de las diferentes especies, el bajo porcentaje de germinación no está asociado a su calidad sino a su estrategia reproductiva. Por ejemplo las especies Baccharis latifolia, Diplostephium rosmarinifolium y Eupatorium angustifolium que tienen una tasa reproductiva alta cuya mortalidad se debe a eventos externos, su tamaño poblacional es variable en el tiempo y su crecimiento es rápido. La estrategia de las especies Bocconia frutescens, Cordia cylindrostachya, Drymis granadensis, Palicourea linearifolia y Palicourea vaginata difiere un poco de las anteriores, puesto que son especies de crecimiento más lento y mayor longevidad, de madera más densa, mayor porte y tasa reproductiva menor. Con base en lo anterior se rechaza la hipótesis propuesta por Hartmann \& Kester (1981), en la que afirman que el bajo porcentaje de germinación y el escaso vigor de las semillas es un comportamiento típico de su baja calidad.

También es posible que el bajo porcentaje de germinación de varias especies se deba a las propiedades genéticas de los individuos de los cuales se tomaron las semillas, o al manejo inadecuado de las mismas en el almacenamiento. Al tratarse de especies a las que no se les ha realizado ningún tipo de estudio se reconoce el desconocimiento de las condiciones ideales de cada una de éstas para manipularlas. Además existen diferencias entre individuos que no se perciben a primera vista y las condiciones en las que se desarrollan varían de un lugar a otro.
Entonces, su supervivencia, desarrollo y mortalidad se puede atribuir a eventos externos y a procesos fisiológicos del medio en el que se desarrollan.

Las especies Cordia cylindrostachya y Palicourea linearifolia muestran graves problemas en su propagación sexual debido al ataque por insectos en sus semillas (familias Notodóntidae y Gelichiidae -Lepidóptera, familia Eulóphidae- Hymenoptera correspondientemente). Este ataque se presenta en las reservas naturales del Distrito Capital de las cuales fue extraído el material reproductivo para realizar la investigación.

Las especies Eupatorium angustifolium y Bocconia frutescens se reprodujeron fácilmente por el método sexual y generaron los mejores resultados frente a las demás especies.

De acuerdo con la escala de Quesada \& Gutiérrez (1972) y adaptada por Segura et al. (1991) la propagación vegetativa de las especies Bocconia frutescens, Cordia cylindrostachya, Palicourea vaginata, Diplostephium rosmarinifolium y Drimys granadensis es muy difícil. Mientras que para la especie Palicourea linearifolia la reproducción por estacas resulta ser de difícil a muy difícil, en Eupatorium angustifolium difícil y en Baccharis latifolia difícil a moderadamente fácil.

Las especies Diplostephium rosmarinifolium y Palicourea vaginata, aunque presentaron porcentajes de sobrevivencia del 27 y $11 \%$, mostraron una tasa de mortalidad del 100 y el $86 \%$; aspecto que limita notablemente su producción, pues son especies sumamente delicadas y no se prestan para reproducir vegetativamente. Por el contrario, las especies Baccharis latifolia, Cordia cylindrostachya, Drymis granadensis, Eupatorium angustifolium y Palicourea linearifolia, presentaron porcentajes de sobrevivencia que oscilan entre el 17 y el 53\%, 
favoreciendo de esta manera el desarrollo de rebrotes y raíces, con valores significativos en la longitud de los primeros.

Los resultados obtenidos en cuanto al comportamiento de las especies ante los tratamientos de AIB, permiten inferir que no existe una misma concentración que favorezca su desarrollo, sino que por el contrario la combinación entre las concentraciones de AIB y el diámetro dió como resultado una respuesta diferente en cada una de las especies de estudio.

Para que las plántulas obtenidas con los tratamientos de AIB se desarrollen favorablemente en el medio natural, es preciso considerar el periodo de mayor producción de rebrotes para transplantarlas y no condicionar crecimiento. De esta manera, las especies Bocconia frutescens, Baccharis latifolia, Eupatorium angustifolium, Palicourea linearifolia, Cordia cylindrostachya y Diplostephium rosmarinifolium deben ser transplantadas entre 25 y 45 días, mientras que las especies Drymis granadensis y Palicourea vaginata deben transplantarse entre 75 y 90 días.

Es necesario resaltar que los procesos de propagación necesitan de cuidado especial en todas sus etapas para obtener resultados favorables. Además, los estudios referentes a la propagación de especies nativas contribuyen enormemente al éxito o fracaso y generan amplias perspectivas de utilización en programas de restauración ecológica.

La evaluación periódica y el registro de las observaciones en cada una de las etapas de proceso de propagación, permiten obtener resultados confiables y seguros acerca del uso de las especies nativas en procesos de restauración ecológica, al mismo tiempo que contribuye en la adquisición de conocimiento acerca de las técnicas de propagación y los aspectos asociados al desarrollo de las especies.

\section{REFERENCIAS BIBLIOGRÁFICAS}

García, F.D. (2001). Protocolo para la propagación vegetativa de las especies Tabebuia rosea (Berthold) DC., y Cordia alliodora (Ruiz \& Pavón) Oken, por medio de propágulos. Tesis de grado, Ingeniería forestal. Universidad Distrital Francisco José de Caldas. Bogotá D. C., Colombia.

Guevara, P \& A. Rocío. 2001. Control de la dormición de semillas de Trompeto (Bocconia frutescens L). Tesis de grado. Universidad Nacional de Colombia, Facultad de Ciencias, Departamento de Biología. Santafé de Bogotá, febrero de

Hartmann,T, Hudson \& Dale E. Kester, 1981. Propagación de plantas, principios y prácticas. Compañía Editorial Continental S. A. México.

ISTA. (1989). Reglas Internacionales para el ensayo de Semillas. Ministerio de Agricultura, Pesca y Alimentación. Madrid España. $P$ 9, 23-24.

Muñetones,O., H. Francy, A. Garcés \& L. Evelyn 2000. Propagación por semillas y estacas de Ocotea calophylla, una estrategia para la conservación de la biodiversidad del ecosistema forestal alto andino. Tesis de grado, Ingeniería forestal. Universidad Distrital Francisco José de Caldas. Bogotá D. C., Colombia.

Niembro, R.A. 1992. Formato descriptivo para la caracterización morfológica de semillas de leguminosas de importancia agroforestal, Revista SEMINA, Año 1, No. 2, México.

Reyes, I. \& J. Oswaldo. 2001. Propagacion vegetativa Tabebuia rosea berthold, Flor morado. Tesis de grado, Ingeniería fores-

Revista Colombia Forestal Vol. 10 No. 20 - Diciembre 2007 
tal. Universidad Distrital Francisco José de Caldas. Bogotá D. C., Colombia.

Segura, A. F. Ariz, R. Martinez, G. Argel,

\& T. Triviño 1991. Propagación agámica de seis especies forestales neotropicales en Colombia, Corporación Nacional de Investigación y Fomento Forestal CONIF, Serie documentación Nº 20, Bogotá, Colombia.

\section{ANEXO}

Anexo 1. Tipos de fruto de las especies en estudio.

\begin{tabular}{|c|c|c|}
\hline Nombre científico & Fruto tipo & Beneficio \\
\hline Baccharis latifolia & $\begin{array}{l}\text { Seco - Inflorescencia } \\
\text { - Capítulo }\end{array}$ & Separar las semillas directamente del capítulo \\
\hline Bocconia frutescens & $\begin{array}{c}\text { Seco - Cápsula } \\
\text { semicarnosa dehiscente }\end{array}$ & $\begin{array}{l}\text { Toman los racimos cuando están completamente maduros y no } \\
\text { tienen las cápsulas abiertas, para estimular luego la apertura de } \\
\text { las mismas secándolas al sol y extraer las semillas }\end{array}$ \\
\hline Cordia cylindrostachya & Carnoso - Drupa & $\begin{array}{l}\text { Tomar los frutos cuando se tornan ligeramente rojizos y luego se } \\
\text { despulpan para obtener la semilla }\end{array}$ \\
\hline Diplostephium rosmarinifolium & Seco - Inflorescencia - Capítulo & Separar las semillas directamente del capítulo \\
\hline Drymis granadensis & Carnoso - Cápsula indehiscente & $\begin{array}{l}\text { Tomar los frutos cuando alcanzan su mayor tamaño } \\
\text { (aprox. } 1.5 \mathrm{~cm} \text { ) y se tornan ligeramente amarillentos, luego se } \\
\text { secan al sol y se extraen sus semillas }\end{array}$ \\
\hline Eupatorium angustifolium & Seco - Inflorescencia - Capítulo & Separar las semillas directamente del capítulo \\
\hline Palicourea vaginata & Carnoso - Baya & $\begin{array}{l}\text { Tomar los frutos cuando se tornan ligeramente azulosos y luego } \\
\text { se despulpan para obtener la semilla }\end{array}$ \\
\hline Palicourea linearifolia & Carnoso - Baya & $\begin{array}{l}\text { Tomar los frutos cuando se tornan ligeramente azulosos y luego } \\
\text { se despulpan para obtener la semilla }\end{array}$ \\
\hline
\end{tabular}

